\title{
Laparoscopic side-to-side esophagogastrostomy using a linear stapler after proximal gastrectomy
}

\author{
Ichiro Uyama ${ }^{1}$, Atsushi Sugioka ${ }^{1}$, Hideo Matsui ${ }^{1}$, Junko Fujita ${ }^{1}$, Yoshiyuki Komori ${ }^{1}$, Yukio Hatakawa ${ }^{2}$, \\ and Akitake Hasumi ${ }^{1}$ \\ ${ }^{1}$ Department of Surgery, Fujita Health University, School of Medicine, 1-98 Dengakugakubo, Kutsukake-cho, Toyoake, Aichi 470-1192, \\ Japan \\ ${ }^{2}$ Hatakawa Clinic, 40-13 Maehama, Hiroishi-cho, Gamagori, Aichi 443-0038, Japan
}

\begin{abstract}
In order to improve anastomotic procedures, we performed laparoscopic side-to-side esophagogastrostomy, using a linear stapler, after proximal gastrectomy in two patients with gastric cancer located in the upper third of the stomach. The patients' postoperative courses were excellent. During postoperative recovery, the patients experienced very little pain, used no analgesic medications, and never experienced reflux esophagitis. This procedure is technically feasible and is an excellent option, given the less involved anastomotic procedure and better postoperative quality of life compared with these features in end-to-side anastomosis using a circular stapler.
\end{abstract}

Key words Laparoscopic surgery - Proximal gastrectomy · Side-to-side anastomosis · Esophagogastrostomy · Linear stapling technique

\section{Introduction}

Proximal gastrectomy is commonly performed for early-stage gastric neoplasms in the upper portion of the stomach. Its frequent use is partially attributable to the improved postoperative fat absorption, improved nutrition, and the release of gut hormones associated with the procedure as compared with these features after total gastrectomy [1-6].

Although laparoscopic distal gastrectomy has been employed extensively, laparoscopic proximal gastrectomy is uncommon, because of the complexity of intracorporeal esophagoenterostomy. Therefore, for the purpose of reducing the complexity of the anastomotic procedure, we performed side-to-side esophagogastrostomy, using linear staplers, as reported by Chassin [7], after performing laparoscopic proximal gas-

Offprint requests to: I. Uyama

Received: February 2, 2001 / Accepted: April 24, 2001 trectomies in two patients with gastric cancer. Here, we describe our surgical procedure and the postoperative courses of these two patients.

\section{Surgical procedure}

Each patient was placed under general anesthesia in the supine, reverse Trendelenburg position with the legs apart. The surgeon stood on the patient's right, with the assisting surgeon on the patient's left, and the camera operator stood between the patient's legs. Pneumoperitoneum was then established, using an open technique, and five working ports (each $12 \mathrm{~mm}$ in diameter) were then placed.

Laparoscopic mobilization and transection of the stomach, and lymphadenectomy (which consisted of a limited dissection A according to the new Japanese practice guidelines for gastric cancer) were performed by the same methods as those that we reported previously (Fig. 1) [8]. In our procedure, in order to prevent pyloric stenosis, postoperative diarrhea, and an increased risk of cholelithiasis, the vagal pyloric, celiac, and hepatic branches were preserved. Accordingly, the esophagus was not transected until after anastomosis, to allow the esophagogastrostomy to be performed safely.

\section{Side-to-side esophagogastrostomy}

This anastomosis involves attaching the posterior aspect of the esophagus to the anterior wall of the gastric remnant. There must be sufficient overlap so that $5-6 \mathrm{~cm}$ of esophagus will lie freely over the front of the gastric remnant. Therefore, the esophagus was sufficiently mobilized to allow this. The posterior wall of the esophagus was excised, and a 1-cm stab wound was made in the anterior wall of the gastric remnant, approximately 5$6 \mathrm{~cm}$ from the staple lines of the stump. A 45-mm endoscopic linear stapler (ETS45; Ethicon Endo-Surgery, 

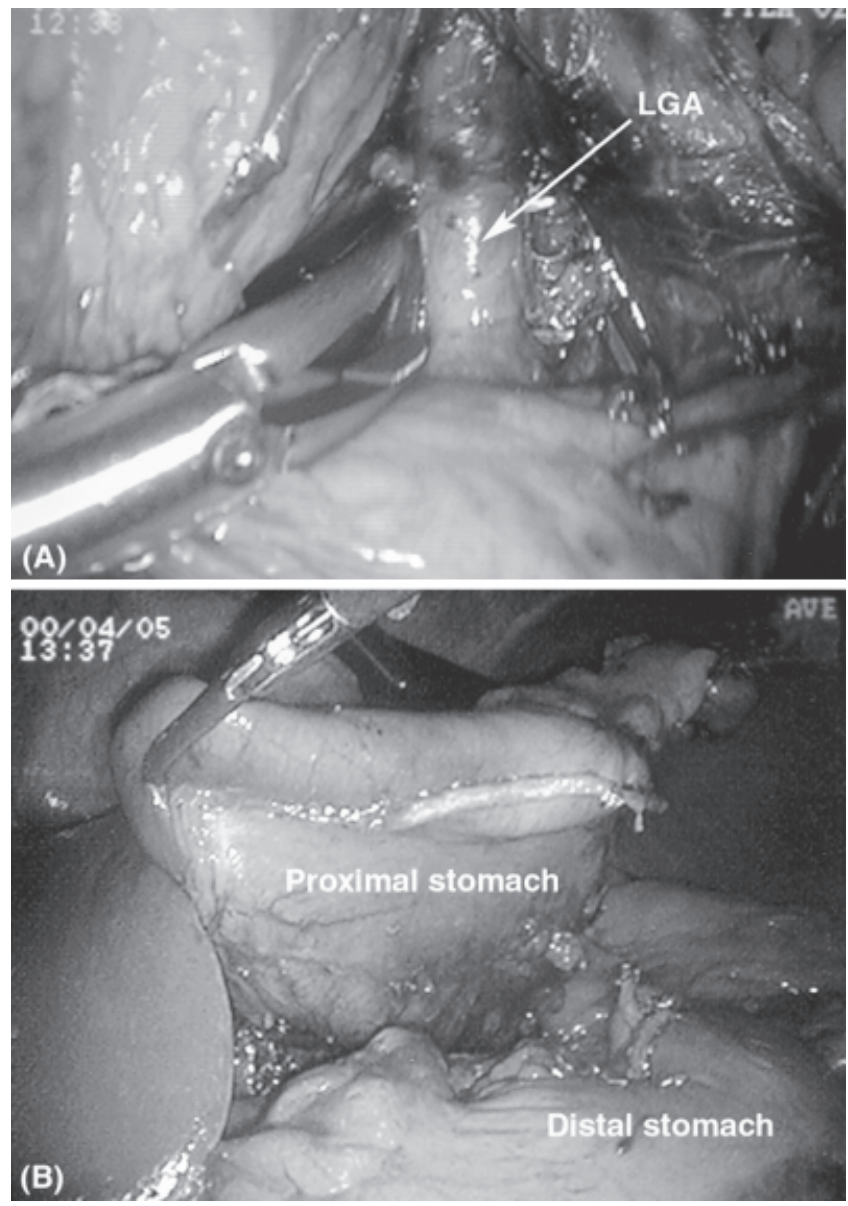

Fig. 1. A The left gastric artery $(L G A)$ was exposed and divided at its origin with double clips, allowing the dissection of the lymph nodes along this artery. B Mobilization and transection of the stomach

Cincinnati, OH, USA) was then used to construct the posterior layer of the esophagogastric anastomosis; this was done by inserting one prong of the stapler into the hole made in the esophagus, and the other prong into the gastric stab wound (Fig. 2). The firing of the stapler converted the two holes into a single entry hole. The esophagus was transected with an ETS45, and the entry hole was closed sequentially, using two ETS45 devices, to construct the anterior layer of the anastomosis (Figs. $3,4)$. The side-to-side esophagogastrostomy and proximal gastrectomy were then completed (Fig. 5). In order to preserve the reservoir function of the gastric remnant, pyloroplasty was not added.

\section{Results}

We performed the side-to-side esophagogastrostomy procedure in two patients with gastric cancers located in the upper third of the stomach. The time required for
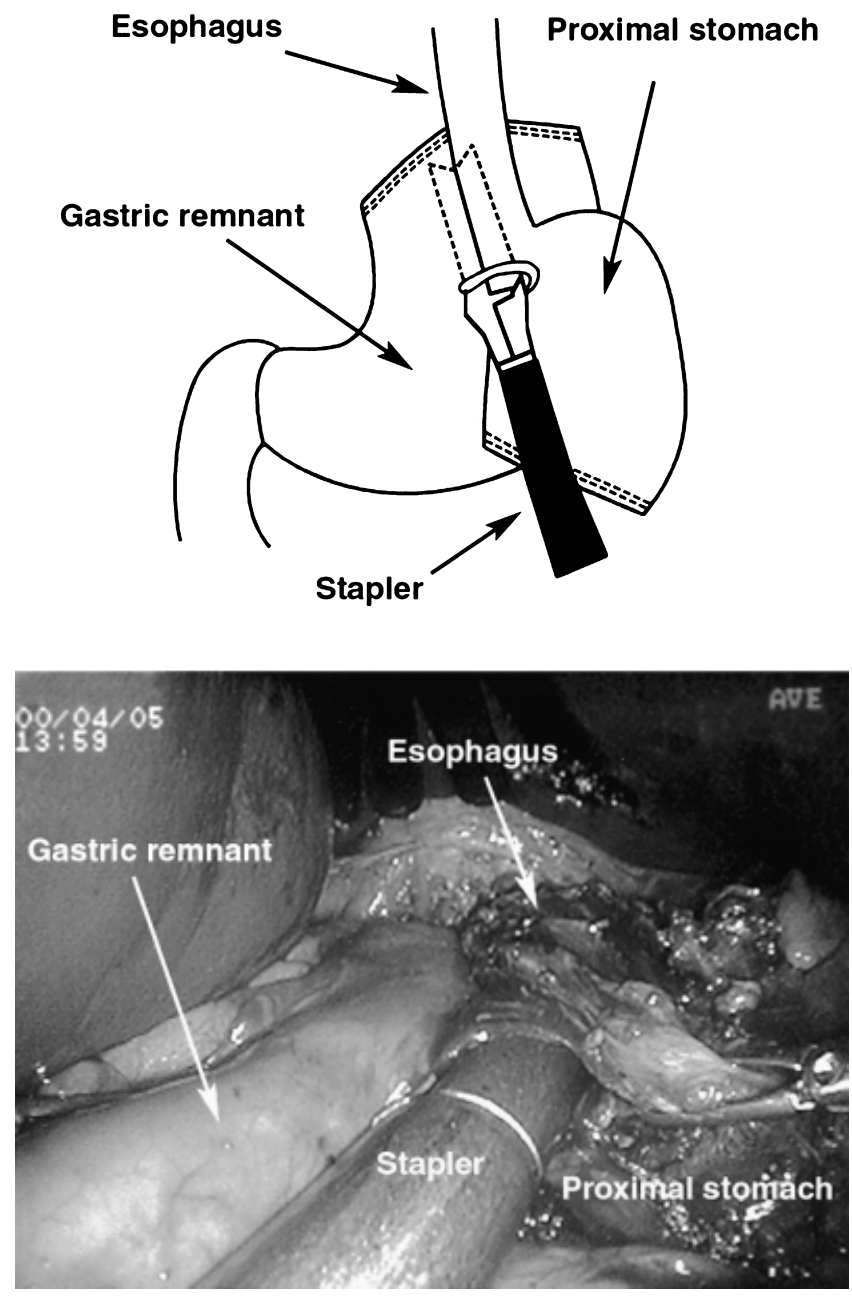

Fig. 2. The posterior wall of the esophagus was excised, and a $1-\mathrm{cm}$ stab wound was made in the anterior wall of the gastric remnant. One prong of the endoscopic stapling device was inserted in the hole made in the esophagus and the other was inserted in the gastric stab wound

this anastomosis was $18 \mathrm{~min}$ in one patient, and $25 \mathrm{~min}$ in the other. In the anastomotic procedure, three ETS45 devices were used. The total operative durations, including the proximal gastrectomy and the anastomosis, were $243 \mathrm{~min}$ and $268 \mathrm{~min}$, respectively. Both patients' recoveries were uneventful, and they returned to normal activities within 12 days. The postoperative hospital stays were 17 days and 24 days, respectively. Neither patient experienced heatburn or stasis. One patient underwent a follow-up gastroscopy and a barium-meal study. The gastroscopy revealed neither reflux esophagitis nor anastomotic stricture. In the barium-meal study performed with the patient in the standing position, we observed gas (similar to a gastric air bubble), an arch-shaped structure of the gastric remnant (which was akin to the normal fornix of the stomach), and a new notch corresponding to the normal cardiac notch (the 

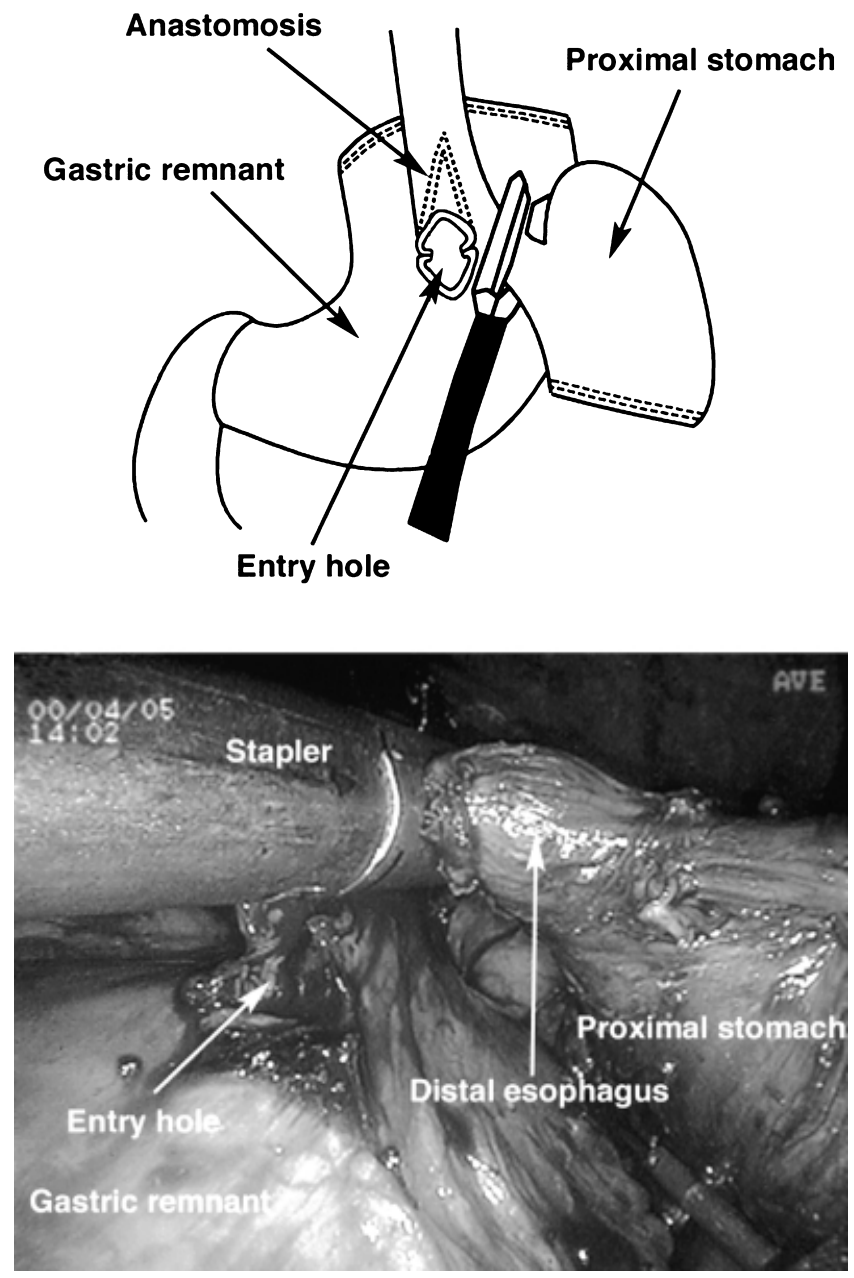

Fig. 3. The firing of the stapler converted the two holes into a single entry hole. The esophagus was transected, using the endoscopic stapling device

angle of His). With the patient in the head-down position, barium regurgitation from the gastric remnant to the esophagus was not shown (Fig. 6).

\section{Discussion}

The standard surgery employed for advanced proximal gastric cancer has been total gastrectomy. For earlystage proximal gastric cancer, proximal gastrectomy has been employed [1-6]. Although laparoscopic distal gastrectomy has recently been widely employed for early gastric cancer [9-12], reports of laparoscopic proximal gastrectomy are rare $[8,13]$. Such procedures are not performed frequently because laparoscopic esophagoenterostomy is technically difficult to perform. We have previously reported a laparoscopic jejunal interposition method as a procedure to prevent postoperative reflux esophagitis in patients with small gastric
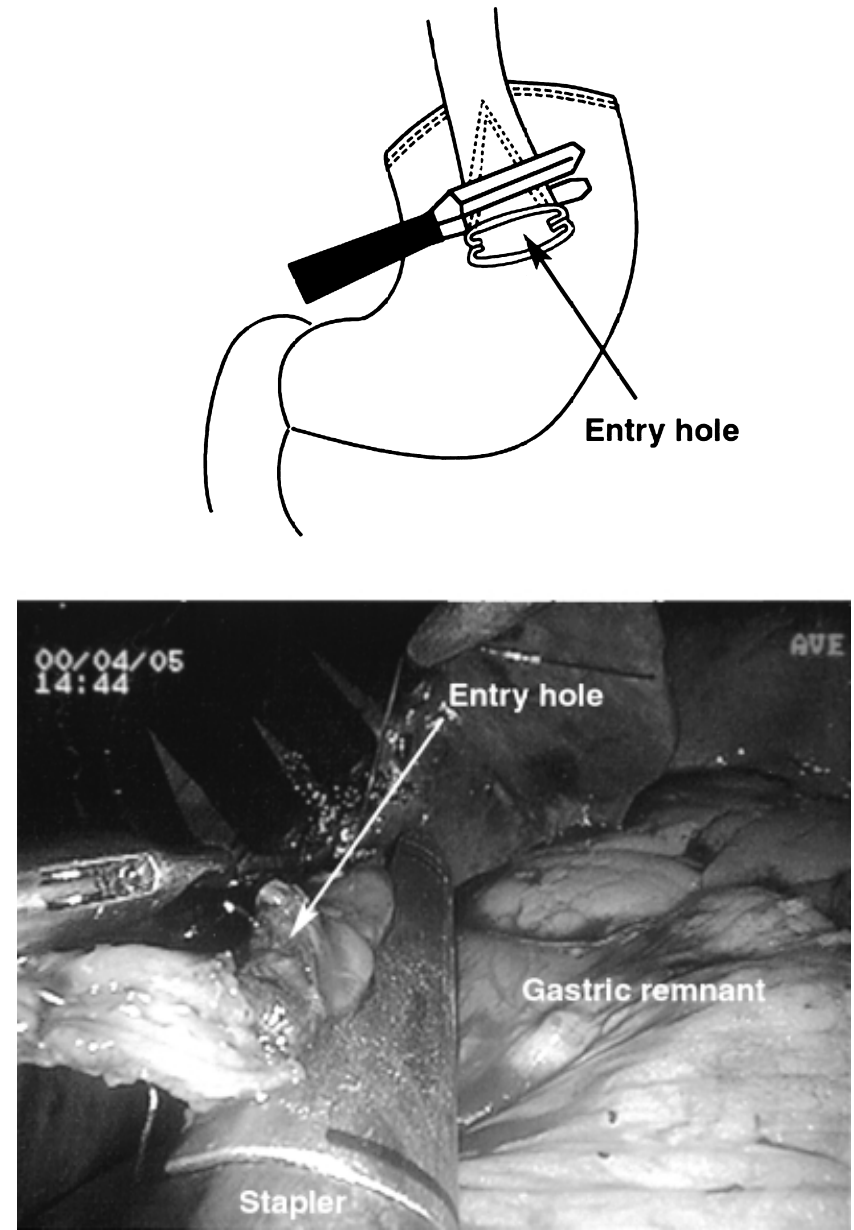

Fig. 4. After the transection of the esophagus, the entry hole was closed to construct the anterior layer of the anastomosis; this was done using two endoscopic stapling devices

remnants [8]. However, this procedure has not been indicated for patients with large gastric remnants, given the following considerations: (1) if jejunal interposition was to be performed for patients with large gastric remnants, postoperative observation of the gastric remnant by endoscopy would sometimes be difficult because of the tortuosity of the interposed jejunum; (2) the procedure is complicated and time-consuming, and requires many stapling devices; and (3) the proximal segment of the jejunum, which is important for nutrient absorption, is scarred by the procedure. Therefore, we have recommended esophagogastrostomy for patients with large gastric remnants. Although end-to-side anastomosis, performed with a circular stapler, is widely employed for esophagogastrostomy, it results in a high incidence of reflux esophagitis. Therefore, in order to prevent regurgitation, it is necessary to add procedures such as wrapping the anastomosis (fundoplication of the Nissen type), making a new notch corresponding to a normal 

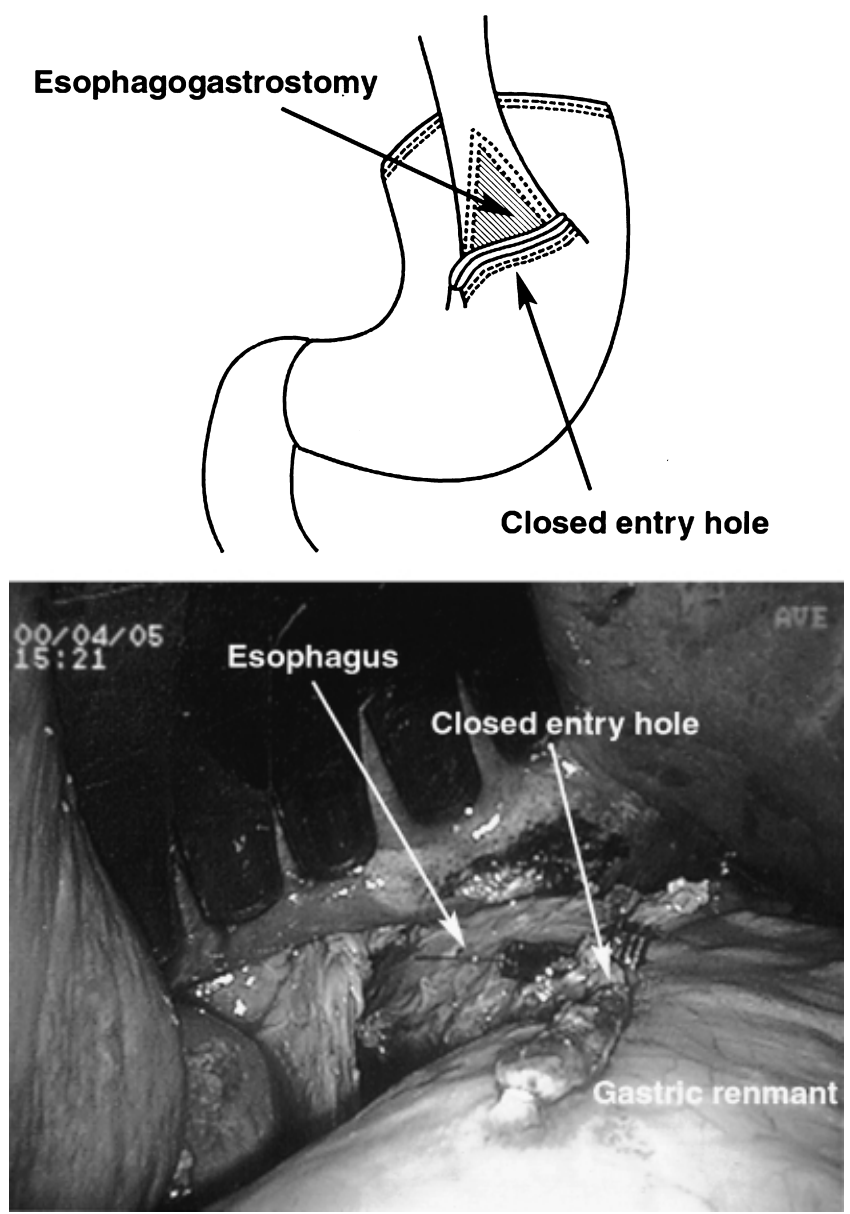

Fig. 5. Appearance of completed, side-to-side esophagogastrostomy

cardiac notch, and forming a flap valve [14-19]. However, such additional procedures are difficult to perform laparoscopically. To reduce postoperative reflux esophagitis and to simplify the anastomotic procedure, Kitano's group [3,13,20] employed gastric tube reconstructions (which have been used after thoracic esophagectomy for esophageal cancer) after laparoscopy-assisted or open proximal gastrectomies. We feel that the procedure used by this group is excellent.

In 1978, Chassin [7] reported a linear stapling technique for esophagogastrostomy after proximal gastrectomy. Although, at present, this technique is uncommon in open surgery, we believed that this linear stapling technique would be efficacious and safe in laparoscopic surgery. Therefore, we decided to attempt a laparoscopic side-to-side esophagogastrostomy, using a linear stapler, after proximal gastrectomy in patients with large gastric remnants. This novel technique has several advantages. (1) The procedure is laparoscopically simple, easy, and safe. (2) Although the lumen size created with the end-to-side anastomosis, using the
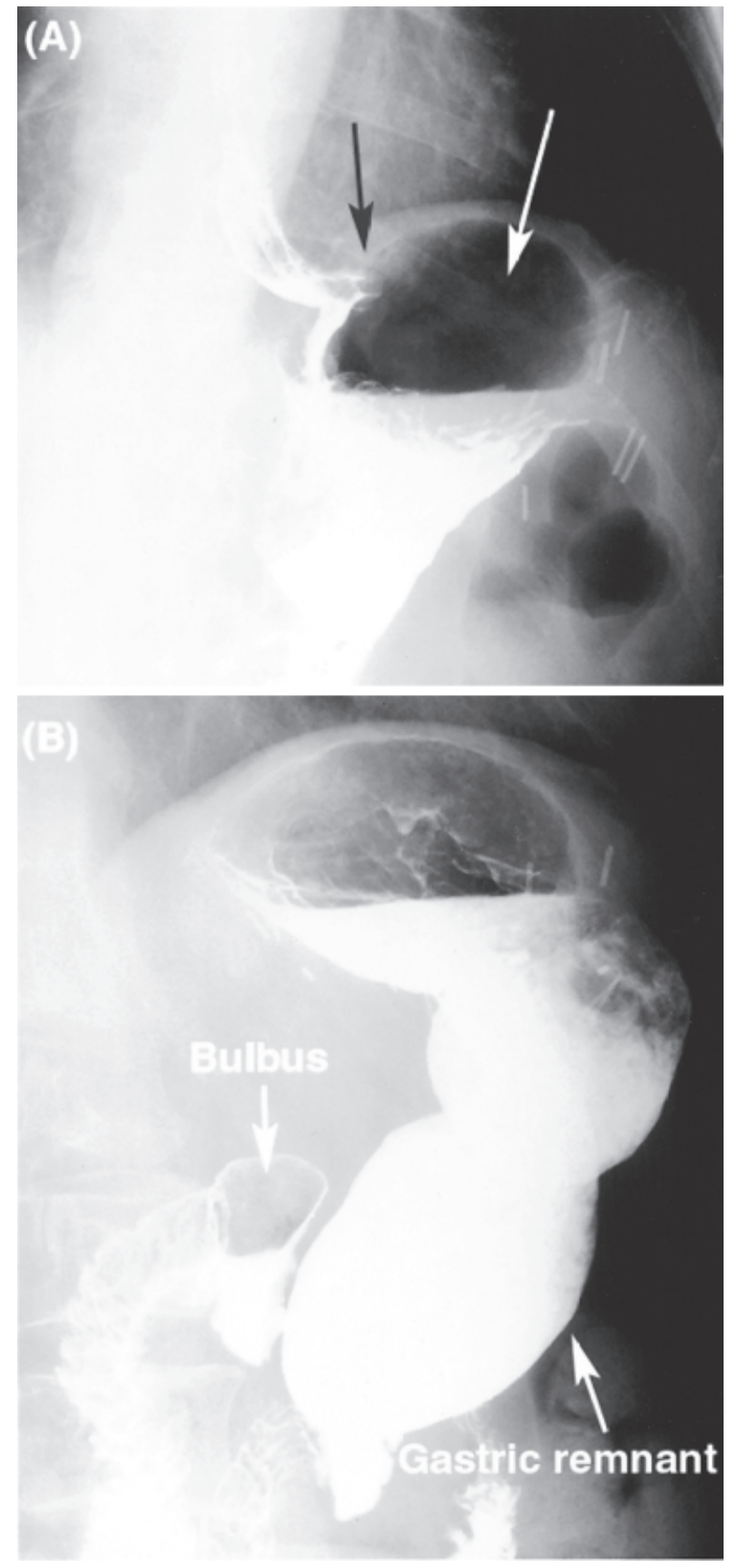

Fig. 6A,B. Postoperative barium-meal study. A Photograph with patient in the standing position. White arrow shows gas (which was similar to a gastric air bubble). The arch-shaped structure of the gastric remnant (which was akin to the normal fornix of the stomach) lies above the anastomosis. The black arrow shows the new notch, which corresponds to the normal cardiac notch (the angle of His). B Photograph with patient in the head-down position. Barium regurgitation from the gastric remnant to the esophagus was not shown

circular stapler, depends on the diameter of the esophagus (with anastomotic stricture sometimes occurring as a complication), the side-to-side anastomosis in our procedure does not depend on the diameter of the esopha- 
gus. Consequently, with our technique, the possibility of anastomotic stricture is small. (3) The proximal segment of the jejunum is preserved.

Our procedure resembles the one reported by Chassin [7], but is original and interesting as an option for those who perform laparoscopic proximal gastrectomies. In patients with a large gastric remnant after a proximal gastrectomy, we recommend side-to-side esophagogastrostomy, using the linear stapler, for laparoscopic surgical procedures.

\section{References}

1. Sawai K, Takahashi T, Suzuki H. New trends in surgery for gastric cancer in Japan. J Surg Oncol 1994;56:221-6.

2. Kaibara N, Nishimura O, Nishidoi H, Kimura O, Koga S. Proximal gastrectomy as the surgical procedure of choice for upper gastric carcinoma. J Surg Oncol 1987;36:110-2.

3. Adachi Y, Inoue T, Hagino Y, Shiraishi N, Shimoda K, Kitano S. Surgical results of proximal gastrectomy for early-stage gastric cancer: jejunal interposition and gastric tube reconstruction. Gastric Cancer 1999;2:40-5.

4. Kameyama J, Ishida H, Yasaku Y, Suzuki A, Kuzu H, Tsukamoto M. Proximal gastrectomy reconstructed by interposition of a jejunal pouch:surgical technique. Eur J Surg 1993;159:491-3.

5. Takeshita K, Saito N, Saeki I, Honda T, Tani M, Kando F, et al. Proximal gastrectomy jejunal pouch interposition for the treatment of early gastric cancer in the upper third of the stomach: surgical techniques and evaluation of postoperative function. Surgery 1997;121:278-86.

6. Siewert JR, Stein HJ, Sendler A, Fink U. Surgical resection for cancer of the cardia. Semin Surg Oncol 1999;17:125-31.

7. Chassin JL. Stapling technique for esophagogastrostomy after esophagogastric resection. Am J Surg 1978;136:399-404.
8. Uyama I, Sugioka A, Fujita J, Komori Y, Matsui H, Hasumi A. Completely laparoscopic proximal gastrectomy with jejunal interposition and lymphadenectomy. J Am Coll Surg 2000;191:114-9.

9. Adachi Y, Shiraishi N, Shiromizu A, Bandoh T, Aramaki M, Kitano S. Laparoscopy-assisted Billroth I gastrectomy compared with conventional open gastrectomy. Arch Surg 2000;135:806-10.

10. Goh PM, So JB. Role of laparoscopic surgery in the management of stomach cancer. Semin Surg Oncol 1999;16:327-31.

11. Uyama I, Sugioka A, Fujita J, Komori Y, Matsui H, Soga R, et al. Completely laparoscopic extraperigastric lymph nodes dissection for gastric malignancies located in the middle or lower third of the stomach. Gastric Cancer 1999;2:186-90.

12. Shiraishi N, Adachi Y, Kitano S, Bandoh T, Katsuta T, Morimoto A. Indication for and outcome of laparoscopy-assisted Billroth I gastrectomy. Br J Surg 1999;86:541-4.

13. Kitano S, Adachi Y, Shiraishi N, Suematsu T, Bando T. Laparoscopy-assisted proximal gastrectomy for early gastric carcinomas. Surg Today 1999;29:389-91.

14. Watkins DH, Prevedel AE, Marper FR. A method of preventing peptic esophagitis following esophagogastrostomy. J Thorac Cardiovasc Surg 1954;28:367-82.

15. Dillard DH, Griffith CA, Merendio KA. The surgical construction of an esophageal valve to replace the cardiac sphincter. An experimental study. Surg Forum 1954;5:306-31.

16. Redo SF, Barnes WA, Sierra OD. Submuscular tunnel in the stomach. Ann Surg 1960;151:37-46.

17. Tocornal JA, Snow HD, Fonkalsrud EW. A mucosal flap valve mechanism to prevent gastroesophageal reflux. Ann Surg 1968; $171 ; 627-37$.

18. Boyd A, Cukingnan R, Engelman RM, Localio SA, Slattery L, Tice DA, Bardin JA, Spencer FC. Esophagogastrostomy. Analysis of 55 cases. J Thorac Cardiovasc Surg 1975;70:817-25.

19. Fisher RD, Brawley RK, Kieffer RF. Esophagogastrostomy in the treatment of carcinoma of the distal two-thirds of the esophagus. Clinical experience and operative methods. Ann Thorac Surg 1972;14:658-70.

20. Adachi Y, Katsuta T, Aramaki M, Morimoto A, Shiraishi N, Kitano S. Proximal gastrectomy and gastric tube reconstruction for early cancer of the gastric cardia. Dig Surg 1999;16:468-70. 